\title{
Adaptive Channel Occupation for Wireless Video Delivery
}

\author{
Ismail Djama and Toufik Ahmed \\ CNRS-LaBRI Lab, University of Bordeaux-1. 351 Cours de la Libération, F-33405 - Talence - France \\ Tel: +33 5400035 47, Fax: +33540003501 \\ E-mail: $\{$ djama,tad $\} @$ labri.fr
}

\begin{abstract}
Bandwidth management in 802.11 wireless networks is becoming the main issue that should be overcome for IP multimedia services delivery such as IPTV, VoD, VoIP, etc.

In this paper, we propose an adaptive bandwidth allocation based on adaptive transmission opportunity to overcome the guaranteed bandwidth issue. This scheme allows the video stream to take up the 802.11 channel proportionally to its throughput by adjusting the channel access priority according to the video transmission rate and the type of video frames (i.e. I, P, or B pictures). In this regards, the 802.11 MAC layer is augmented with an application-awareness that exploits RTP-level information for grouping and transmitting video frames. Performance evaluations using an experimental test-bed show the capability of our proposal to guarantee bandwidth for video streaming application while reducing considerably packet video loss compared to a conventional system.
\end{abstract}

Index-terms: Packet Video, QoS, TXOP, Streaming, 802.11, Cross-Layer Design.

\section{INTRODUCTION}

During this last decade, the 802.11 wireless networks have evolved rapidly from novelty into necessity technology for the last mile connection. The 802.11 WLAN is used currently for delivering all IP services such as data, voice, video, and multimedia. However, the wireless transmission undergoes the QoS issue that should be considered for wide acceptance of these services by end-users. Maintaining the QoS continuity for multimedia streams over 802.11 networks is challenging due to some intrinsic wireless transmission properties such as fast fading, co-channel interferences, and shadowing. The extra-overhead introduced by the 802.11 medium access control such as long headers size, inter-frame spaces, back-off time, and acknowledgments [1] make the situation more difficult. The bandwidth guarantee is also an issue as the channel is shared among different concurrent streams of different natures and requirements.

To cope with wireless transmission characteristics, the 802.11 specification [2] proposes some mechanisms to improve the transmission performance and reliability such as MAC frame retransmission using a limited number of retry and MAC fragmentation. With the same focus, others mechanisms were proposed by different studies such as the link rate adaptation by switching among different modulations and channel coding [3] and adjusting the transmission power. The overhead introduced by the 802.11 MAC layer was also tackled by new mechanisms such as frame concatenation [4], piggyback [4], and frame grouping [5].

However, when used alone, the above mentioned mechanisms aim to increase the reliability of transmission without any consideration for application-level traffic characteristics, type of service, and quality of service required per stream. Many research works have started to consider a joint cooperation between the service level, network level, and data link level to improve some aspect of the QoS. In this context, the cross layer design has emerged as new paradigm to allow an interlayer communication and configuration for QoS continuity and provisioning [6]. The cross-layering is generally considered as an improvement of the layered architecture by defining new interfaces at each layer to allow the collaboration between the adjacent and non adjacent layers.

This paper fills in the category of cross-layer design for video QoS provisioning over 802.11 wireless networks. Thus, we propose a bandwidth control scheme based on adaptive transmission opportunity (TXOP) to overcome the guarantee bandwidth issue for real-time video packets delivering. The proposed mechanism allows the video stream to take up the 802.11 channel proportionally to its throughput by adjusting the access time according to the video throughput and the type of the video frame being delivered (i.e. I, P, or B pictures).

Different cross-layer techniques proposed in the literature were focused on MAC and physical (PHY) layers interactions in order to adjust 802.11 MAC mechanisms according to the wireless channel state. In our approach, we propose an application/MAC level interaction by exploiting in-band signalling information presents at RTP-level header. For this purpose, the 802.11 MAC layer is augmented with an application-awareness which allows it to send the MAC frames belonging to the same video picture in burst mode without a new contention round. However, the contention is maintained between video pictures to avoid the channel monopolization (for example: passing from I to $\mathrm{P}$ picture).

The remainder of this paper is organized as follow: Section II introduces the overhead and the bandwidth management issues in the 802.11 link layer. Section III details our proposed bandwidth control scheme based on adaptive frame group size (i.e. Adaptive TXOP). Section IV presents performance evaluations of the proposed scheme and details the conducted experiments. Finally, section V concludes the paper. 


\section{THE OVERHEAD AND THE BANDWIDTH MANAGEMENT IN 802.11 LINK LAYER}

It is generally known that the transmission performances are degraded in wireless network in terms of bandwidth. This means that there is a big difference between the rate provided by the physical layer and the rate that can be achieved by the application layer. The difference is caused mainly by the time overhead introduced by the 802.11 link layer mechanisms for each transmitted frame. At the physical level, this time corresponds to the transmission of the physical preamble (PLPC preamble and PLCP header) which is generally transmitted with low bit rate (1Mbits/s or $2 \mathrm{Mbits} / \mathrm{s})$. At the MAC level, this time includes the Inter Frame Spacing (IFS) waiting intervals and the backoff time which assure the channel access fairness in the Distributed Coordination Function (DCF).

Thus, the total overhead time introduced by the 802.11 link access called $t_{\text {overhead }}$ can be calculated according to the equation Eq.1. It includes the Distributed IFS (DIFS) interval $t_{\text {DIFS }}$, the backoff time $t_{\text {backoff }}$, the transmission time of the physical header of the frame $t_{\mathrm{PHY} \text { header }}$, the transmission time of the MAC header $\mathrm{t}_{\mathrm{MAC} \_ \text {header }}$, the Shortest IFS (SIFS) interval $t_{\text {SIFS }}$, the reception time of the physical header of the acknowledgment $t_{\text {ack_PHY header, and the reception time of the }}$ acknowledgment $t_{\text {ack}}$. It is important to notice that the RTS/CTS frames are not considered in this equation.

$$
\begin{gathered}
t_{\text {overhead }}=t_{\text {DIFS }}+t_{\text {backoff }}+t_{P_{\text {PHY }} \text { header }}+t_{\text {MAC_header }} \\
+t_{\text {SIFS }}+t_{\text {ack_PHY_header }}+t_{\text {ack }}
\end{gathered}
$$

For a particular physical rate, the $t_{\text {overhead }}$ value depends mainly on the $t_{\text {DIFS }}$ and the $t_{\text {backoff. }}$ In contrast, the $t_{\text {DIFS }}$ value depends on the channel state while the $t_{\text {backoff }}$ fluctuates randomly and depends on the success or the failure of the last transmissions.

In order to reduce the $t_{\text {overhead }}$ which represents the main issue for the performance degradation (rate, delay, and jitter) in the wireless access networks, many mechanisms have been proposed during the last years: the frame concatenation [4], the frame piggybacking [4], and the frame grouping [5].

The frame concatenation mechanism consists of grouping in one MAC packet data unit all the service data units that have the same destination by adding a small concatenation header. This allows sharing the $\mathrm{t}_{\text {overhead }}$ among different service data units. However, this mechanism is limited in providing QoS guarantee due to frame corruption during the wireless transmission since the corruption probability increases by increasing the number of concatenated frames. Consequently, the number of service data units should be limited and should be determined according to the wireless channel conditions and traffic characteristics.

The frame piggybacking mechanism is useful in an active bidirectional communication between two stations. It allows the receiving station to send its data frames within the acknowledgements packets returned to the sender. This allows the station to save the access channel time by $t_{\text {DIFS }}+t_{\text {backoff }}$. However, this mechanism does not provide any advantage for one way communication such as video streaming.
In this paper, we are more interested to the frame grouping mechanism which allows sharing $t_{\text {DIFs }}+t_{\text {backoff }}$ time interval among different MAC frames having the same destination. The benefit of this mechanism relays on MAC frames transmission in burst mode without releasing the channel. To keep the control over the channel, the successive MAC frames transmissions are separated by SIFS interval which is shorter than DIFS. The frame grouping is inspired from the fragmentation burst introduced by the 802.11 specification when the MAC fragmentation is employed. In this configuration, all fragments belonging to the same MAC service data unit are sent in burst without a new contention for each fragment. Figure 1 illustrated the difference between the frame grouping transmission and the standard transmission.

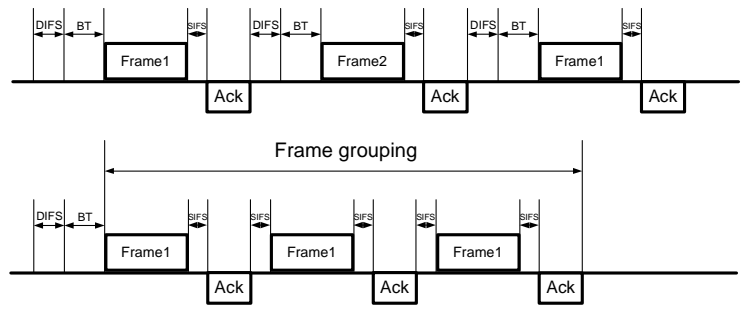

Figure 1: The frame grouping (down figure) compared to a standard transmission (top figure)

The frame grouping has many advantages: (1) it avoids the frames corruption by avoiding increasing the frame size transmitted over the wireless channel compared to frame concatenation mechanism, (2) it shares the channel access time among many frames, and (3) it allows bandwidth control by guaranteeing the rate for the delivered data at the link access level since different frames can be sent once the channel is obtained.

The frame grouping mechanism is used in IEEE 802.11e standard. This last improves the media access control layer of IEEE 802.11 to satisfy different QoS requirements by introducing two channel access functions: the enhanced distributed channel access (EDCA) and the Hybrid Coordination Function (HCF) Controlled Channel Access (HCCA). In Enhanced Distributed Channel Access (EDCA) model, each Access Category (AC) has a particular transmission opportunity (TXOP) that represents a time interval in which the AC has the capability to send in burst many frames in the same way as the frame grouping. Table 1 resumes the TXOP default values proposed by the IEEE 802.11e standard.

\begin{tabular}{|c|c|c|c|c|c|}
\hline \multicolumn{2}{|c|}{ Classe } & $\begin{array}{c}\text { AC_BK } \\
\text { Background }\end{array}$ & $\begin{array}{c}\text { AC_BE } \\
\text { Best Effort }\end{array}$ & $\begin{array}{c}\text { AC_VI } \\
\text { VIdeo }\end{array}$ & $\begin{array}{c}\text { AC_VO } \\
\text { VOice }\end{array}$ \\
\hline \multirow{2}{*}{$\begin{array}{c}\text { TXOP } \\
\text { (ms) }\end{array}$} & FHSS & 0 & 0 & $6.016 \mathrm{~ms}$ & $3.264 \mathrm{~ms}$ \\
\cline { 2 - 6 } & DSSS & 0 & 0 & $3.008 \mathrm{~ms}$ & $1.504 \mathrm{~ms}$ \\
\hline
\end{tabular}

Table 1 : TXOP values in the IEEE 802.11e standard

Regarding the HCCA mechanism, the TXOP is specified for each QSTA (QoS station) according to the TSPEC (Traffic specification) transmitted to the QAP (QoS Access Point).

To allow bandwidth guarantee for real-time streaming applications, we have employed an adaptive frame grouping mechanism leading to an adaptive TXOP interval. Our 
mechanism allows sending MAC frames belonging to the same video frame in burst without a new contention round.

However, an important challenge is arisen when the frame grouping mechanism is used : How to delimitate the time interval during which the frames can be sent in burst? This time interval should achieve two mains goals: (1) guarantying the bandwidth in correlation with the application throughput and (2) avoiding the channel monopolization when different frames are concatenated.

To overcome this challenge, we have made the frame grouping mechanism independent from the time in comparison with 802.11e standard. Indeed, instead of fixing the TXOP interval for each $\mathrm{AC}$, we propose to determine the number of frames that should be sent in burst mode. This allows our mechanism to be independent from the physical rate. To illustrate this purpose, let us take the 802.11e access category AC_VI which is defined specially for video transmission. In this AC, the TXOP interval is equal to $3.008 \mathrm{~ms}$ (see Table 1). With an average frame size equal to 1512 bytes and a physical rate equal to $2 \mathrm{Mbits} / \mathrm{s}$, only 4 frames can be sent in burst. However, when the physical rate increases to $11 \mathrm{Mbits} / \mathrm{s}, 22$ frames can be sent in burst.

Adaptive TXOP is not a new solution in 802.11 networks. In [7][8], an adaptive transmission opportunity scheme is proposed. It allows the station to have a rate adaptation capability by dynamically configuring its TXOP limit within the maximum TXOP value of IEEE 802.11e. In [9], the authors propose modifying the TXOP interval dynamically based on the packets in the queue, so that fragments of the same packet are sent in the same TXOP interval. However, this mechanism is dependent on the physical rate of the channel. All the above mentioned research works and many others do not consider the traffic characteristics (i.e. type of the delivered video pictures: I, P or B), and the current traffic throughput (i.e. support of Variable Bit Rate "VBR" video streaming) as proposed by our scheme.

Therefore, our mechanism determines the relevant number of MAC frames that forms a TXOP interval rather than fixing its value (as done by 820.11 e). These MAC frames create a group and the challenge is to determine the size of this group to allow bandwidth guarantee. To reach this goal, intensive experiments have been performed to compare different strategy of frames group size ranging from fixed (i.e. 802.11e) to adaptive. This study will be presented in next Sections.

\section{ADAPTIVE FRAMES GROUP SIZE}

To illustrate the proposed bandwidth guarantee mechanism based on MAC-level video frame grouping, Figure 2 shows an example of an adaptive frames group size taking a video Frame (i.e. I, P, B Pictures) as a group delimiter. Picture $m$ and Picture $m+1$ create two separate groups.

In Our proposal, we define a group as a video picture (or Video Frame) which is broken into multiple RTP video packets at the application level framing. The RTP packet size depends on the maximum transfer unit of network (approximately 2301 bytes for 802.11 and 1500 bytes for Ethernet LAN) in order to avoid the fragmentation at the network and MAC layer. The number of RTP packets generated from one video picture depends mainly on the video throughput and the picture type (i.e. I, P, or B pictures) used in the MPEG coding.

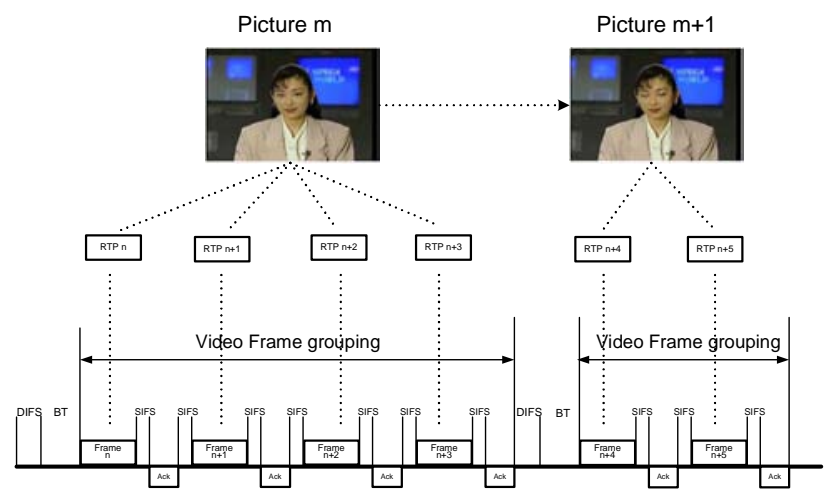

Figure 2 : Bandwidth guarantee based on MAC-level Video Frame Grouping

It is clear that the number of RTP packets increases proportionally according to the video throughput. For a particular video throughput, the number of RTP packets in I-picture is higher than P-picture which is higher than B-picture. Table 2 illustrates the average number of RTP packets (1450 octets) per picture type coded at different throughput of the same MPEG-4 video sequence.

\begin{tabular}{llccc} 
& & \multicolumn{3}{c}{ Number of RTP packets } \\
\hline Video throughput & I & P & B \\
\hline $\mathbf{5 0 0}$ & Kbits/s & 7 & 2 & 1 \\
$\mathbf{1}$ & Mbits/s & 12 & 5 & 2 \\
$\mathbf{1 . 5}$ & Mbits/s & 16 & 7 & 3 \\
$\mathbf{2}$ & Mbits/s & 20 & 10 & 5 \\
$\mathbf{2 . 5}$ & Mbits/s & 20 & 12 & 6 \\
$\mathbf{3}$ & Mbit/s & 24 & 14 & 7 \\
\hline
\end{tabular}

Table 2 : Average number of RTP packets per video picture

Using a video picture as a group delimiter in the frame grouping mechanism provides a dynamic frame group size. This latter will be not fixed as proposed by $802.11 \mathrm{e}$ but it will be rather dependent on video throughput and will be independent from the physical rate (e.g. [9]). This allows a correlation between the number of MAC frames sent in burst and the video throughput at the application level. The mechanism avoids the channel monopolization since after each video picture transmission the sender should contend with concurrent streams to gain the access to wireless channel with the same priority.

The frame grouping mechanism is performed at the 802.11 MAC layer by using application-level information. It is MAC centric approach in which the MAC layer is augmented with an application-awareness to recognize a video picture at the MAC level. For this purpose, the MAC layer exploits the inband RTP signalling information available in the MAC frame payload. The RTP header provides timestamp information which can be used to distinguish among different video picture. The timestamp reflects the sampling instant of the first 
byte in the RTP data packet. All RTP packets belonging to the same video picture should have the same timestamp since they belong to the same sampling time. When the timestamp differs for two successive MAC frames, then a new video picture is detected and the MAC layer should contend for accessing the channel before transmission. This introduces some traffic fairness among different sessions.

\section{Performance Evaluation}

In this section, we present the performance evaluation of the bandwidth guarantee mechanism based on adaptive frame group size. For this evaluation, we performed real experiments using an experimental test-bed. The proposed mechanism is implemented in Madwifi driver [10] which is an open-source driver for wireless cards based on Atheros chipset. Figure 3 shows the experimental test-bed composed of one 802.11 Access Point (AP) and two wireless stations (STA1 and STA2). The physical rate of the 802.11 network is limited to $5.5 \mathrm{Mbit} / \mathrm{s}$. STA1 transmits video stream to the AP using VLC software [11]. The STA2 does not use the frame grouping mechanism and it transmits to the AP a UDP or TCP concurrent streams using Iperf software [12].

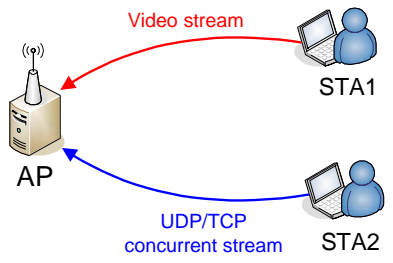

\section{Figure 3 : Experimental test-bed}

The performance evaluations are organized in two parts. In the first part, we compare the adaptive frame group size to a fixed group size (i.e 802.11 TXOP). In the second part, we assess the capability of our mechanism to grantee the bandwidth of video stream with different rates for the concurrent stream.

For the first part of experiments, we used two video sequences (type: MPEG-4, resolution: 352x288, structure: IBBPBBPBBPBB, $25 \mathrm{fps}$ ). Table 3 gives the rest of the two video characteristics.

\begin{tabular}{|c|c|c|}
\hline & Video 1 & Video 2 \\
\hline Throughput average & $860 \mathrm{Kbits} / \mathrm{s}$ & $1252 \mathrm{Kbits} / \mathrm{s}$ \\
\hline $\begin{array}{c}\text { Average packet number for } \\
\text { I-Picture }\end{array}$ & 11 & 14 \\
\hline $\begin{array}{c}\text { Average packet number for } \\
\text { P-Picture }\end{array}$ & 4 & 6 \\
\hline $\begin{array}{c}\text { Average packet number for } \\
\text { B-Picture }\end{array}$ & 2 & 3 \\
\hline
\end{tabular}

Table 3 : Video characteristics

Using these two video sequences, we have performed a comparative study according to the test plane described in Table 4. Each test was repeated 10 times and averages were calculated form the obtained results.

\begin{tabular}{|c|c|c|c|}
\hline Test & Grouping strategy & Video & UDP (STA2) \\
\hline Test1 & Without Frame grouping & \multirow{5}{*}{ Video 1} & \multirow{5}{*}{$900 \mathrm{Kbits} / \mathrm{s}$} \\
\hline Test2 & Group Size $=2$ & & \\
\hline Test3 & Group Size $=3$ & & \\
\hline Test4 & Group Size $=5$ & & \\
\hline Test5 & $\begin{array}{l}\text { Adaptive Frame Group Size (our } \\
\text { proposal) }\end{array}$ & & \\
\hline Test6 & Without Frame grouping & \multirow{4}{*}{ Video 2} & \multirow{4}{*}{$500 \mathrm{Kbits} / \mathrm{s}$} \\
\hline Test7 & Group Size $=3$ & & \\
\hline Test8 & Group Size $=5$ & & \\
\hline Test9 & $\begin{array}{l}\text { Adaptive Frame Group Size (our } \\
\text { proposal) }\end{array}$ & & \\
\hline
\end{tabular}

Table 4: Tests Plane

Figure 4 and Figure 5 show respectively the average throughput and the average loss achieved by the transmission of video 1. Figure 6 and Figure 7 show the same results for video 2 . From these results, we can see clearly that a fixed group size can't satisfy the need of video throughput. For example, the group size equal to 3 frames allows the video 1 to get a sufficient bandwidth at the wireless channel since the video 1 doesn't undergo packet loss (Figure 5). However, the same group size doesn't allow the video 2 to take up the required bandwidth at the wireless channel to cancel the loss (Figure 7). In the other side, the proposed solution (Test 5 for video 1 and Test 9 for video 2) adapts the channel occupation according to the video throughput and cancel the loss for the two videos sequences.

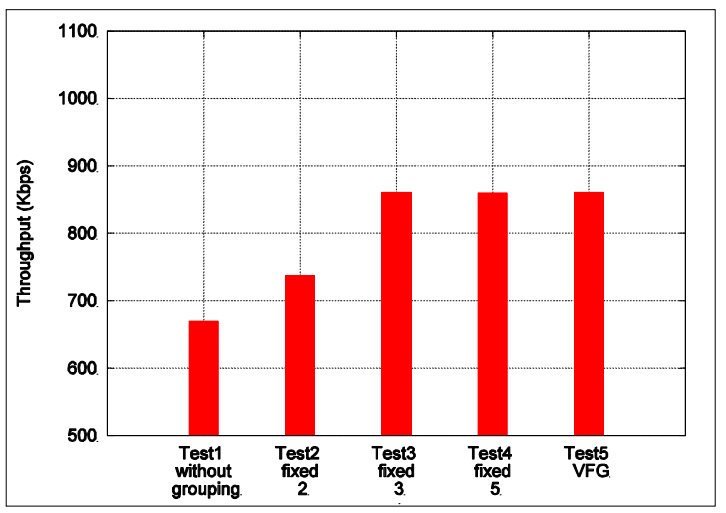

Figure 4 : Average throughput for test1 to test5 of video 1

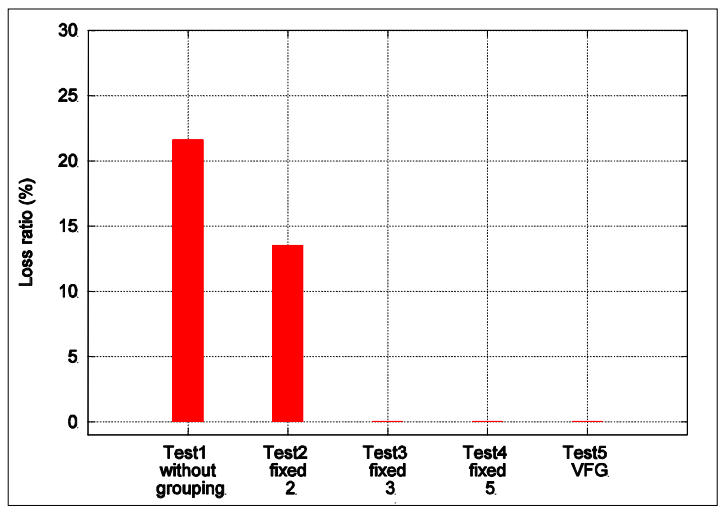

Figure 5 : Average loss ratio for test1 to test5 of video 1 


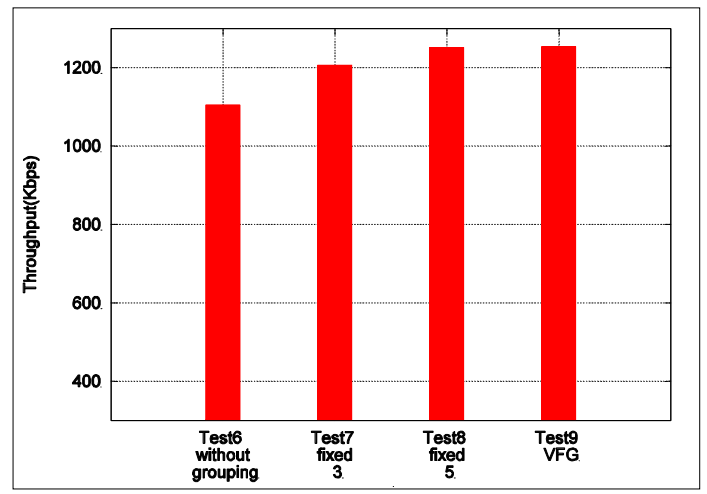

Figure 6 : Average throughput for test6 to test9 of video 2

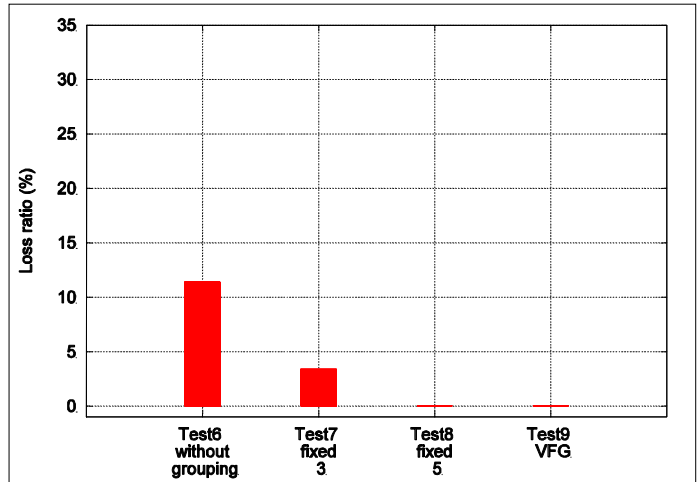

Figure 7 : Average loss ratio for test6 to test9 of video 2

In the second part of experiments, we evaluate the performance of our bandwidth guarantee scheme with concurrent raw-UDP streams transmitted by STA2 with different constant bit rates: $0.5 \mathrm{Mbit} / \mathrm{s}, 1 \mathrm{Mbit} / \mathrm{s}, 1.5 \mathrm{Mbit} / \mathrm{s}, 2$ Mbit/s, 2.5 Mbit/s, $3 \mathrm{Mbit} / \mathrm{s}$. For each rate, we perform two scenarios:

- Scenario 1: We use a standard system without any bandwidth guarantee at the STA1

- Scenario 2: Bandwidth guarantee scheme is employed at STA1

The characteristics of the video sequence (type: MPEG-4, resolution: 352x288, structure: IBBPBBPBBPBB, $25 \mathrm{fps}$ ) transmitted by the STA1 are resumed in Table 5.

\begin{tabular}{|c|c|}
\hline & Video \\
\hline Throughput average & $2279 \mathrm{Kbits} / \mathrm{s}$ \\
\hline $\begin{array}{c}\text { Average packet number for } \\
\text { I-Picture }\end{array}$ & 20 \\
\hline $\begin{array}{c}\text { Average packet number for } \\
\text { P-Picture }\end{array}$ & 11 \\
\hline $\begin{array}{c}\text { Average packet number for } \\
\text { B-Picture }\end{array}$ & 6 \\
\hline
\end{tabular}

Table 5 : Video characteristics

Figure 8 (resp. Figure 10) and Figure 9 (resp. Figure 11) depict respectively the average loss ratio and the received average throughput for video stream (resp. raw UDP streams) for each test with a specific transmitted UDP rate presented at the $\mathrm{x}$-axis.

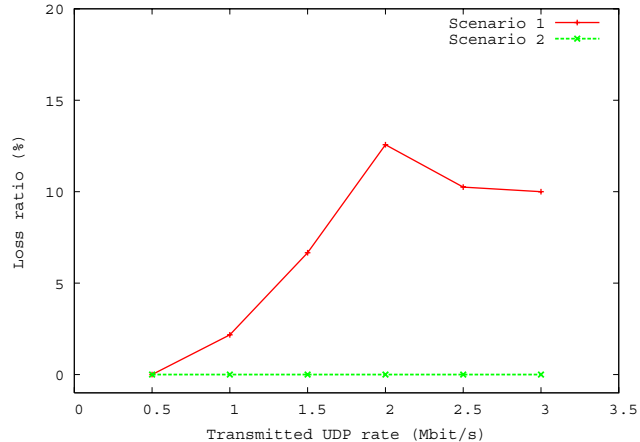

Figure 8 : Average loss ratio for video stream

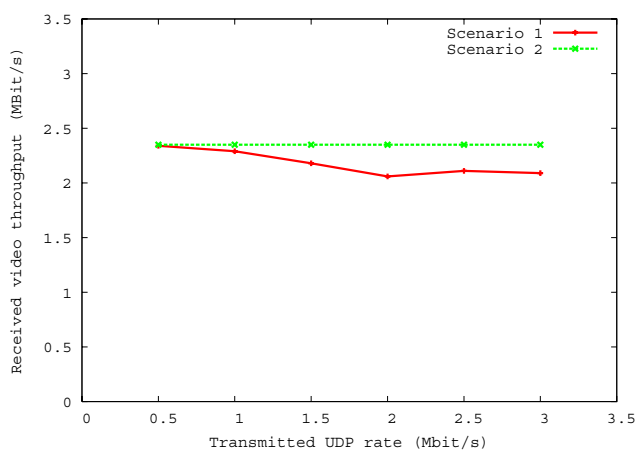

Figure 9 : Received average throughput for video stream

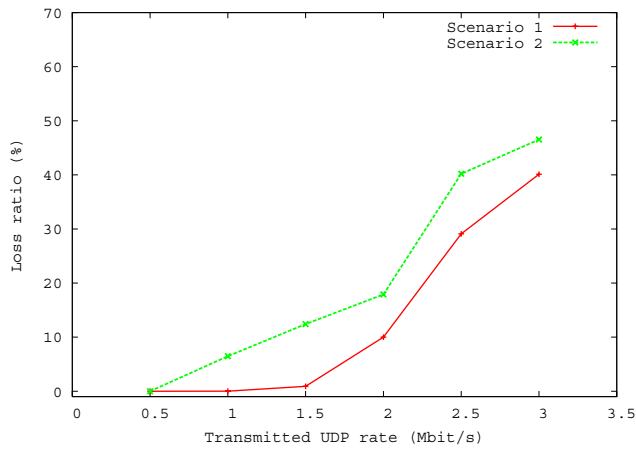

Figure 10 : Average loss ratio for raw-UDP stream

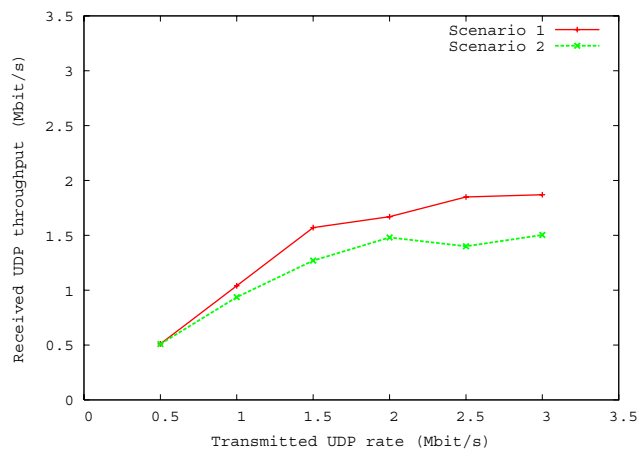

Figure 11 : Received average throughput for raw-UDP stream

With a concurrent UDP stream of $0.5 \mathrm{Mbit} / \mathrm{s}$, we see in these results that both raw-UDP and video stream are transmitted correctly without any loss. However, the difference between the two scenarios can be noticed with raw-UDP rate of $1 \mathrm{Mbit} / \mathrm{s}$ and more. In scenario 1 , video loss ratio increases (see Figure 8) and its received throughput decreases (see Figure 9), while the raw-UDP stream doesn't undergo loss until 1.5 Mbit/s (see Figure 10 and Figure 11). However, in scenario 2, 
we can notice that video throughput is guaranteed at the expense of raw-UDP stream. Thus, loss ratio for raw-UDP stream is higher in scenario 2 compared to scenario 1 (Figure 10). We can see also the consequent on the received throughput compared to transmitted throughput in the network (Figure 11). Therefore, it is clear from these results that our scheme increases bandwidth guarantee for video stream by adjusting the channel occupation according to the video transmission rate and independently from the concurrent stream rate.

The same results were obtained with a concurrent TCP streams which are summarized in Table 6.

\begin{tabular}{|c|c|c|c|c|}
\hline & \multicolumn{2}{|c|}{ Scenario 1 } & \multicolumn{2}{c|}{ Scenario 2 } \\
\hline & $\begin{array}{c}\text { TCP } \\
\text { stream }\end{array}$ & $\begin{array}{c}\text { Video } \\
\text { Stream }\end{array}$ & $\begin{array}{c}\text { TCP } \\
\text { stream }\end{array}$ & $\begin{array}{c}\text { Video } \\
\text { Stream }\end{array}$ \\
\hline Loss ratio (\%) & $/$ & $25.1 \%$ & $/$ & $0 \%$ \\
\hline $\begin{array}{c}\text { Throughput } \\
\text { (Mbit/s) }\end{array}$ & $1.88 \mathrm{Mbit} / \mathrm{s}$ & $1.74 \mathrm{Mbit} / \mathrm{s}$ & $\begin{array}{c}1.28 \\
\mathrm{Mbit} / \mathrm{s}\end{array}$ & $\begin{array}{c}2.35 \\
\mathrm{Mbit} / \mathrm{s}\end{array}$ \\
\hline
\end{tabular}

Table 6: Tests results with concurrent TCP stream

\section{CONCLUSION}

In this work, we have proposed a bandwidth guarantee scheme which allows enhancing the priority of the video stream over 802.11 wireless networks. Our mechanism is implemented at MAC-layer and it is traffic-aware. It allows sending a group of MAC frames belonging to a particular video picture in burst mode. Performance evaluations of the proposed mechanism for video streaming service show a considerable enhancement of the overall received video quality in term of bandwidth guarantee and packet loss reduction. Furthermore, our scheme avoids the monopolization of the wireless channel access by releasing the channel after sending the particular group. The results show the added value of our proposal comparing with a fixed group size (TXOP in 802.11e). They show also the effect of our proposal on UDP and TCP concurrent streams for providing traffic differentiation compared to $802.11 \mathrm{e}$ model.

\section{REFERENCES}

[1] Y. Xiao and J. Rosdahl, "Throughput and delay limits of IEEE 802.11,'IEEE Commun. Lett., vol. 6, no. 8, pp. 355-357, Aug. 2002.

[2] IEEE 802.11, IEEE Standards for Information Technology -Specific Requirements -- Part 11: Wireless LAN Medium Access Control (MAC) and Physical Layer (PHY) Specifications, Edition (ISO/IEC 8802-11: 1999), 1999.

[3] Haratcherev, J. Taal, K. Langendoen, R. Lagendijk and H. Sips, "Automatic IEEE 802.11 rate control for streaming applications", Wireless Communications and Mobile Computing, Vol 5, pp.412-437, 2005.

[4] Yang Xiao, "IEEE 802.11 performance enhancement via concatenation and piggyback mechanisms", IEEE Transactions on Wireless Communications, Volume 4, Issue 5, Page(s): 2182 - 2192, sept 2005.

[5] J.Tourrilhes, "Packet frame grouping: improving IP multimedia performance over CSMA/CA", IEEE International Conference on Universal Personal Communications ICUPC'98, Volume 2, Page(s):1345 - 1349 vol.2, Oct 1998.
[6] S. Shakkotai, T. Rappaport, P. Karlsson, "Cross-layer design for wireless networks", IEEE Communications Magazine, vol.41, no.10, pp. 74-80, October 2003.

[7] EunKyung Kim; Young-Joo Suh "ATXOP: an adaptive TXOP based on the data rate to guarantee fairness for IEEE 802.11e wireless LANs", in IEEE VTC2004, Vol. 4, pp. 2678 - 2682, Fall. 2004

[8] Min Li Huang, Seungbeom Lee, Sin-Chong Park "An adaptive EDCA TXOP with rate adaptation for QoS provision", in 6th WSEAS International Conference on Instrumentation, Measurement, Circuits and Systems, pp. 13-18 2007.

[9] Bheemarjuna Reddy T., John John P, Siva Ram Murthy C. "Providing Mac Qos For Multimedia Traffic In 802.11e Based Multi-Hop Ad Hoc Wireless Networks" In Computer Networks, Vol. 51, Issue 1, Pp. 153 - 176, January 2007.

[10] Multiband Atheros Driver for WIFI available at http://madwifi.org/

[11] VideoLan open source project available at http://www.videolan.org/vlc/

[12] Iperf tool available at http://dast.nlanr.net/Projects/Iperf/ 